\title{
Decameter Type III-Like Bursts
}

\author{
V.N. Melnik ${ }^{1}$, A.A. Konovalenko ${ }^{1}$, B.P. Rutkevych ${ }^{1}$ \\ H.O. Rucker ${ }^{2}$, V.V. Dorovskyy ${ }^{1}$, E.P. Abranin ${ }^{1}$, \\ A. Lecacheux ${ }^{3}$, A.I. Brazhenko ${ }^{4}$, A.A.Stanislavskyy ${ }^{1}$

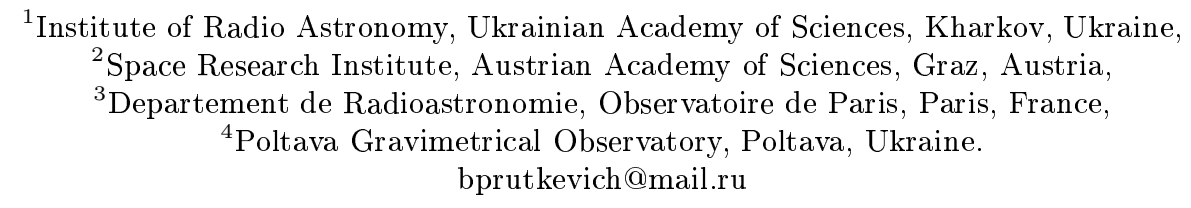

Starting from 1960s Type III-like bursts (Type III bursts with high drift rates) in a wide frequency range from 300 to $950 \mathrm{MHz}$ have been observed. These new bursts observed at certain frequency being compared to the usual Type III bursts at the same frequency show similar behaviour but feature frequency drift 2-6 times higher than the normal bursts. In this paper we report the first observations of Type III-like bursts in decameter range, carried out during summer campaigns 2002 - 2004 at UTR-2 radio telescope. The circular polarization of the bursts was measured by the radio telescope URAN-2 in 2004. The observed bursts are analyzed and compared with usual Type III bursts in the decameter range. From the analysis of over 1100 Type III-like bursts, their main parameters have been found. Characteristic feature of the observed bursts is similar to Type III-like bursts at other frequencies, i.e. measured drift rates $(5-10 \mathrm{MHz} / \mathrm{s})$ of this bursts are few times larger than that for usual Type III bursts, and their durations (1-2 s) are few times smaller than that for usual Type III bursts in this frequency band.

\section{Introduction}

For the first time Type III-like bursts were observed in the frequency range $500-950 \mathrm{MHz}$ [6]. Their frequency rates were higher than $2000 \mathrm{MHz} / \mathrm{s}$ and sometimes even infinite. The author also reported bursts with reverse drifts (from low to high frequencies). The observed number of Type III-like bursts was $20 \%$ of normal Type III bursts. Durations of fast bursts ranged from $0.3 \mathrm{~s}$ to $2 \mathrm{~s}$. According to [6], their radio fluxes had values from 5 s.f.u. to 50000 s.f.u. There was a tendency that the faster Type III-like bursts had shorter duration.

In paper [5] it was found that Type III-like bursts appeared mainly in the frequency band $400-800 \mathrm{MHz}$, but sometimes this band was as wide as 200-950 MHz. There was information [2] that Type III-like bursts were observed at frequencies $40 \mathrm{MHz}$ and $60 \mathrm{MHz}$.

Detailed analysis of properties of Type III-like bursts observed at frequencies 310-340 MHz was performed in [3, 4]. It was found that 50\%, $17 \%$ and 33\% of Type III-like bursts out of 402 analyzed ones had forward, reverse and infinite drift rates, respectively. The mean duration of Type III-like bursts was $0.26 \mathrm{~s}$ which is $1 / 4$ of that for usual meter range Type III bursts. Paper [3] showed that frequency drift rates and durations of these bursts depended on the position of the active region associated with these bursts on the solar disk. From this fact the author concluded that propagation effects play a significant role in generation of these bursts.

An attempt to find Type III-like bursts in decameter range was reported in paper [7], where the authors analyzed the storm observed at UTR-2 radio telescope on June, 8-9, 1977. Authors concluded that if such bursts existed they had drift rates and durations close to that for usual Type III bursts.

In this paper we present results of observations of decameter (10-30 MHz) Type III-like bursts at radio telescope UTR-2 performed in 2002-2004 and URAN-2 in 2004, we also analyze their parameters and discuss their properties. 


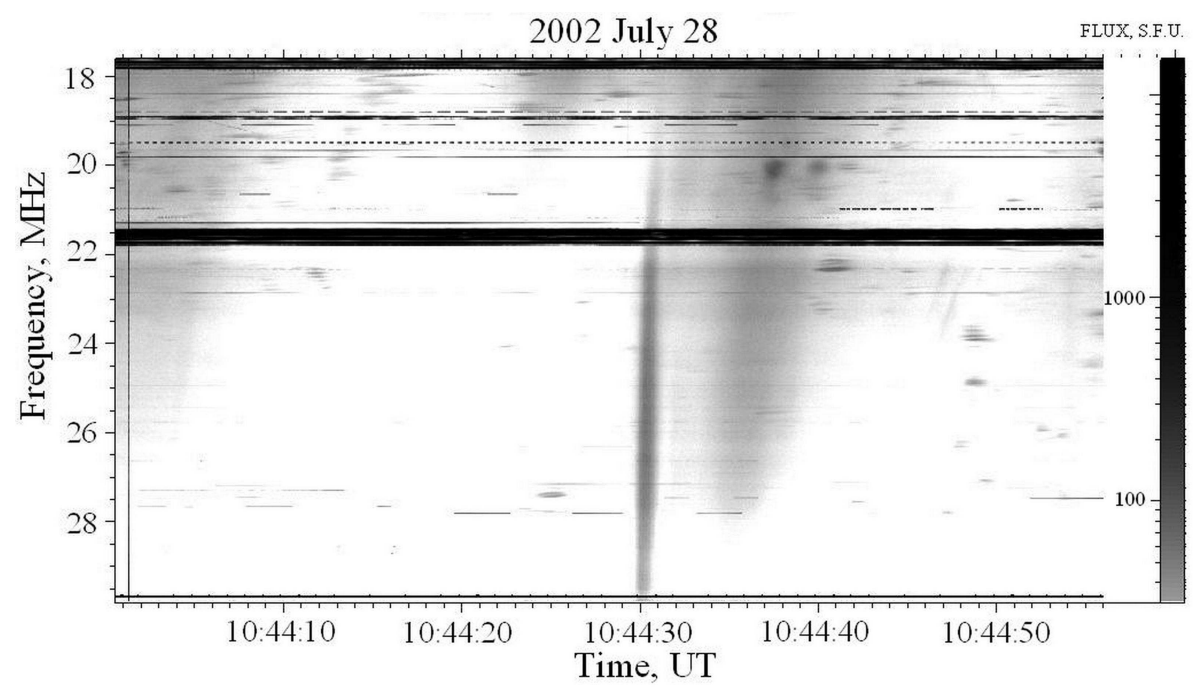

Figure 1: Dynamic spectrum of Type III-like burst. Three bright vertical areas at 10:43:40, 10:44:35 and 10:45:00 correspond to normal Type III bursts, and a narrow bright line at 10:44:30 indicates a Type III-like burst.

\section{Observations}

Type III-like bursts reported in this paper were registered at radio telescope UTR-2 during summer campaigns of 2002-2004 years. Three sections of the radio telescope, with effective area of $30000 \mathrm{~m}^{2}$ providing the beam of $1^{\circ} \times 13^{\circ}$ were used. In 2002 the registrations were carried out by a Digital Spectrum Polarimeter (DSP) with frequency resolution $12 \mathrm{kHz}$, time resolution $20 \mathrm{~ms}, 50 \mathrm{~ms}$ and $100 \mathrm{~ms}$, and sensitivity $5 \mathrm{Jy}$. The frequency band in these observations was $12 \mathrm{MHz}$. The measurements in 2003-2004 were carried out by a 60-channel spectrometer with frequency resolution $3 \mathrm{kHz}$, and time resolution of $10 \mathrm{~ms}$ at frequency range 10-30 MHz.

According to the observations in the decameter range $(10-30 \mathrm{MHz})$, the usual Type III bursts have frequency drift rates of 2-4 MHz/s and durations of $4-10 \mathrm{~s}$. Besides these bursts we registered fast Type III bursts, with drift rates exceeded, sometimes considerably, those for typical Type III bursts. Following [3], these fast bursts can be called as Type III-like bursts. In this report we consider the bursts observed in 2002 (July 13-17, July 26-30 and August 16-19), 2003 (July 1-6), and in 2004 (June 17-22).

The total time of observations was approximately 120 hours and there were detected about 1100 Type III-like bursts. All bursts had negative frequency drift rates (from high to low frequencies). Decameter Type III-like bursts similarly to their high frequency analogs were shorter than usual Type III bursts (Figure 11), and their time profiles were symmetrical (Figure 21). There were fast bursts with fine frequency structure similar to Type IIIb bursts, which we call Type IIIb-like bursts. The fastest Type III-like burst was observed on August 18, 2002 with the drift rate of about $40 \mathrm{MHz} / \mathrm{s}$.

\section{Analysis}

The big number of observed bursts allows statistical analysis of Type III-like bursts. The distribution of Type III-like bursts according to their frequency drift rates is shown in Fig. 3a. The majority of bursts have frequency rates $12 \mathrm{MHz} / \mathrm{s}$, though there are bursts with essentially larger rate values.

One can see, that in the decameter range the drift rate of fast bursts is few times bigger than that for usual bursts. A similar relation between the fast and normal Type III bursts was observed at higher frequencies [3, 4, 5].

The typical duration of decameter Type III-like burst is $1-2 \mathrm{~s} \mathrm{(Fig.} \mathrm{3b),} \mathrm{which} \mathrm{is} \mathrm{about} 1 / 4$ of the average duration of ordinary decameter Type III bursts, their flux values are in the range 10 to 60 s.f.u. (Fig. 4a). We analyzed 5 storms and found that the majority of Type III-like bursts had been observed when the active regions associated with them were located near the central solar meridian, according to the data from Pulkovo Observatory of Russian Academy of Sciences [8]. For example in Fig. 4b the occurrence frequency 


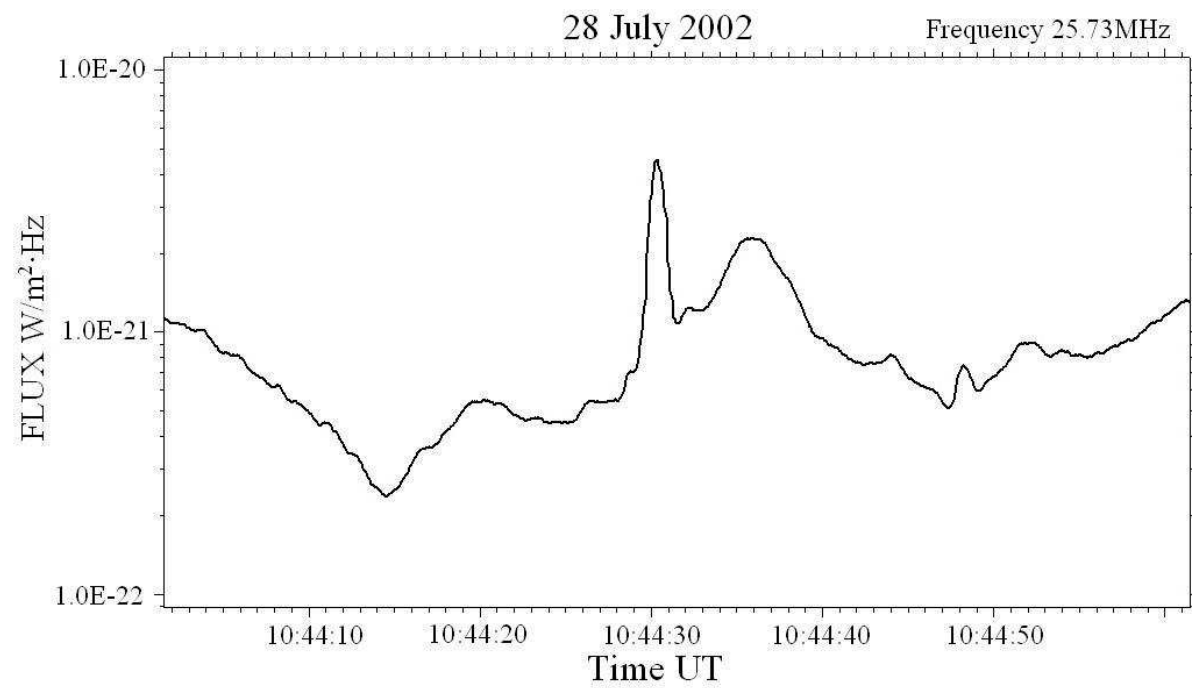

Figure 2: Time profiles of Type III-like burst at the frequency $22.11 \mathrm{MHz}$. Noticeable symmetrical shape.
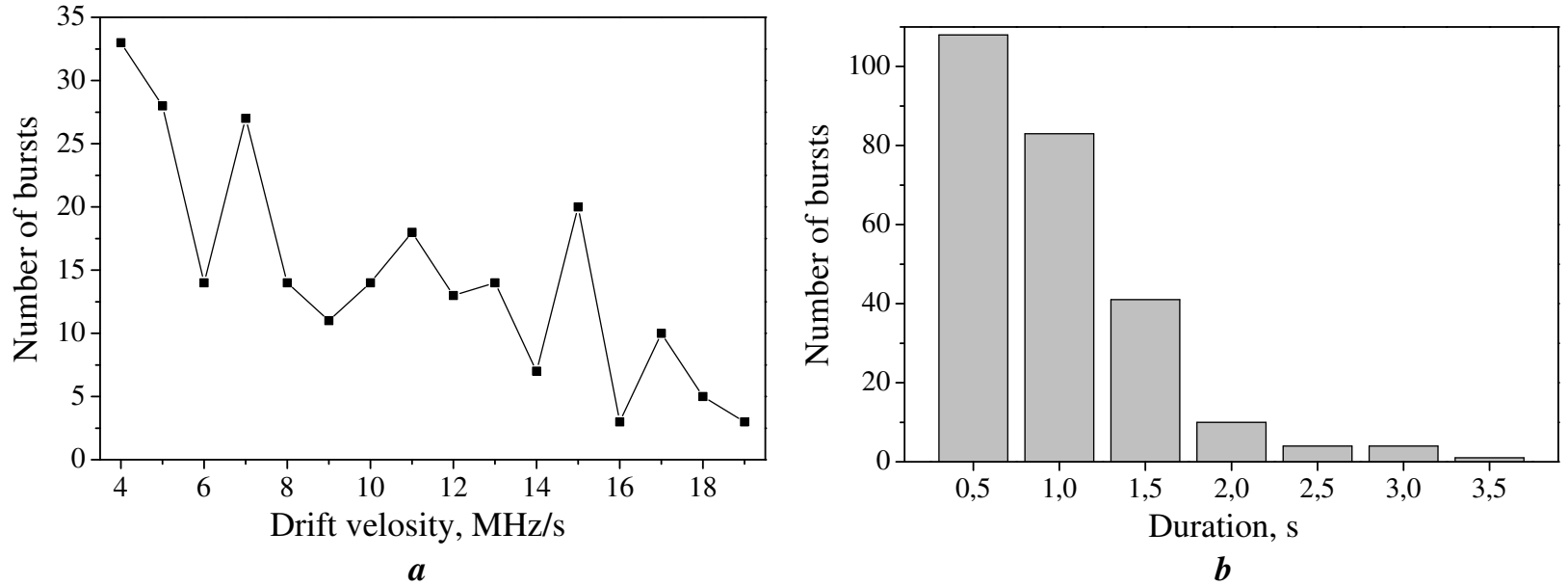

Figure 3: (a)The frequency drift rate and, (b) duration distribution of Type III-like bursts, observed in July 2003. 


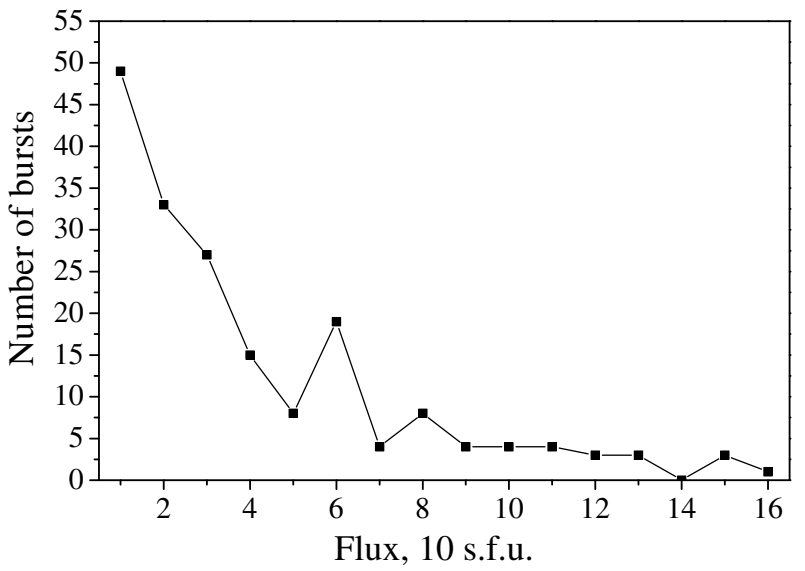

$a$

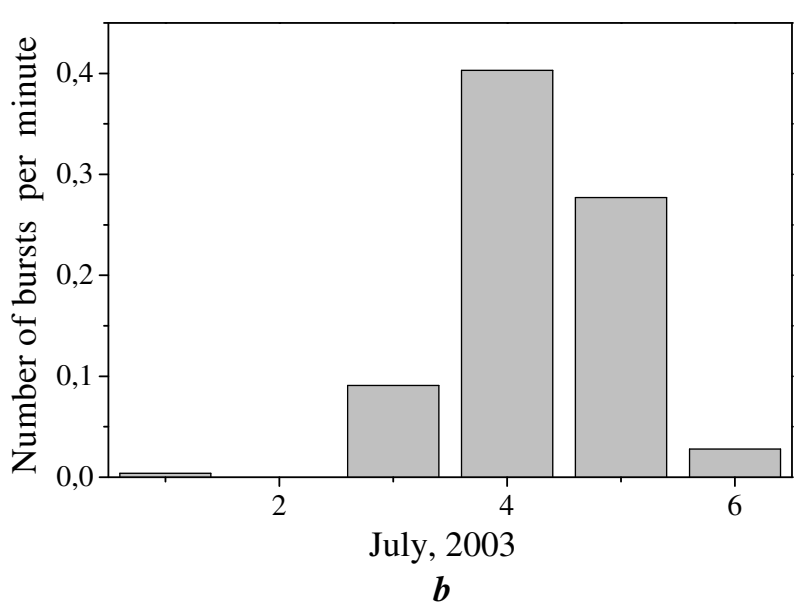

b

Figure 4: (a) The flux distribution of Type III-like bursts, observed in July 2003, and (b) the appearance rate of Type III-like bursts during five days from 1st to 6th of July 2003.

of Type III-like bursts within duration of one storm is shown. The maximum on 4 July corresponds to the day, when the active region associated with the Type III-like bursts was near to the central meridian. An even more interesting case of two overlapping peaks on a similar frequency occurrence diagram was observed when two active regions on the Sun disk appear within a short time interval.

During the storm of June 17-22, 2004, the measurements of polarizations were made for a part of Type III-like bursts by means of radio telescope URAN-2 [1. The polarization degree of Type III-like bursts proved to be equal to 10-15\%. There were also observed Type III-like bursts with fine frequency structure similar to usual Type IIIb bursts. Such Type III-like bursts were not observed at higher frequencies, they are typical for the decameter range only.

Up to now, all kinds of Type III and Type III-like bursts were explained by the gradients of plasma density in the solar corona. According to some models, the fast Type III-like bursts in the high frequency range appear due to the fact that they are generated in the low corona, where the density profile is steeper [3, 6]. Following this model, the observed high frequency drift rates of decameter Type III-like bursts forces us to suppose that such density gradient is even higher in the corona, and such irregularities must occupy very large regions (comparable with the solar radius). However there is no evidence for existence of such high density gradients in the corona. This leaves unanswered the question about generation of big number of the Type III-like bursts in the decameter range. The dependence of appearance rate of Type III-like bursts via active region position suggests that the propagation effects of electromagnetic waves in the solar corona should be of big importance in this process.

\section{Conclusions}

For the first time we discovered the Type III-like bursts in decameter range. These bursts show properties similar to those for Type III-like bursts observed at higher frequencies. For example, their durations are $1 / 4$ of usual decameter Type III bursts durations, and their drift rates are few times of those for the usual decameter Type III bursts. The number of Type III-like bursts correlates with the position of the active region associated with them. According to the polarization measurements of these bursts (approximately $10-15 \%$ ), one can conclude that they seem to be generated at the second harmonic of the plasma frequency. The existing model of high frequency Type III-like bursts assume that the plasma density gradients are much higher in the low corona. Such assumption cannot be applied to the decameter bursts. The total number of Type III-like bursts in the decameter range is remarkably high (up to one bursts per 2.5 minutes), therefore they cannot be explained by occasional extraordinary variations of solar parameters, and there should exist a model based on typical values of solar parameters.

Taking into account significant similarity between Type III-like bursts at high frequency observed before and recently observed decameter Type III-like burst (in particular the correlation between the position of the active region on the solar disk and the burst occurrence rate) it may appear that the suggested model of 
high frequency Type III-like burst should be improved.

\section{References}

[1] Brazhenko A.I., Bulatsen V.G., Vashchishin R.V., et al. KFNTS.-2005. Suppl., V. 5, p.43 (2005)

[2] Gopala Rao U.V. Austral.J.Phys., V. 18, p.283 (1965)

[3] Elgaroy O. A\&A, V. 82, p.308 (1980)

[4] Elgaroy O., Rosenkilde H. Coronal Disturbances IAU Symp., V. 57, p.249 (1974)

[5] Kundu M.R., Roberts J.A., Spencer C.L., Kuiper J.W. ApJ, V. 133, p.255 (1961)

[6] Young C.W., Spencer C.L., Moreton, G.E. Roberts, J.A. ApJ, V. 133, p.243 (1961)

[7] Zaitsev V.V. and Levin B.N. A\&A, V. 134, p.268 (1984)

[8] http://www.gao.spb.ru/ 